\title{
Erratum
}

\section{Leptin-regulated gene expression in MCF-7 breast cancer cells: mechanistic insights into leptin-regulated mammary tumor growth and progression}

\author{
Candida N Perera ${ }^{1}$, Hwei G Chin ${ }^{1}$, Nadire Duru ${ }^{1}$ and Ignacio G Camarillo ${ }^{1,2}$ \\ ${ }^{1}$ Department of Biological Sciences, ${ }^{2}$ Purdue Cancer Center, Purdue University, West Lafayette, Indiana 47907, USA \\ (Correspondence should be addressed to I G Camarillo; Email: ignacio@bilbo.bio.purdue.edu)
}

The authors and the journal apologise for errors that have occurred in the above article which appeared in the November issue of the Journal of Endocrinology volume 199.
Errors have occurred in Table 3 on page 226 and references were omitted, the correct table is published below.

Table 3 Leptin-regulated genes identified by microarray when compared with previous reports. Listed in the table below are leptin-regulated genes that were determined by microarray, which have been previously identified. The effect of leptin measured by microarray is compared with the results of prior reports, with the type of cell previously evaluated included.

Published results (references)

\begin{tabular}{|c|c|c|c|c|}
\hline & Result & Cell/tissue type & Reference & Our findings \\
\hline \multicolumn{5}{|l|}{ Gene } \\
\hline CDK2 & $\uparrow$ by Leptin & Human MCF-7 cells & Okumura et al. (2002) & $\uparrow$ by Leptin \\
\hline MAP2K1 & $\uparrow$ by Leptin & Human T47-D cells & Laud et al. (2002) & $\uparrow$ by Leptin \\
\hline STAT1 & $\uparrow$ by Leptin & Mouse adipose tissue & Bendinelli et al. (2000) & $\uparrow$ by Leptin \\
\hline FOS & $\uparrow$ by Leptin & Human placental cells & Cauzac et al. (2003) & $\uparrow$ by Leptin \\
\hline EPOR & $\downarrow$ by Leptin & Rat T-cells & Nakatsuji (2006) & $\downarrow$ by Leptin \\
\hline Kiss1 & $\downarrow$ by Leptin & Mouse neuron cells & Smith et al. (2006) & $\downarrow$ by Leptin \\
\hline CTGF & $\uparrow$ by Leptin & Human NRK-49F cells & Lee et al. (2004) & $\uparrow$ by Leptin \\
\hline \multirow[t]{4}{*}{ Cyclin D1 } & $\uparrow$ by Leptin & Human MCF-7 cells & Okumura et al. (2002) & $\uparrow$ by Leptin \\
\hline & $\uparrow$ by Leptin & Human T-47D \& MDA-MB-231 cells & Ray et al. (2007) & \\
\hline & $\uparrow$ by Leptin & Human MCF-7 cells & Hu et al. (2002) & \\
\hline & $\uparrow$ by Leptin & Rat hepatic stellate cells & Saxena et al. (2004) & \\
\hline \multirow[t]{2}{*}{ CDKN1B (p27) } & $\downarrow$ by Leptin & Mouse pancreatic $\beta$ cells & Uchida et al. (2005) & $\downarrow$ by Leptin \\
\hline & & Mouse CD4 T cells & De Rosa et al. (2006) & \\
\hline TGFB1 & $\uparrow$ by Leptin & Rat glomerular endothelial cells & Han et al. (2001) & $\uparrow$ by Leptin \\
\hline \multirow[t]{2}{*}{ IGF1R } & $\uparrow$ by Leptin & Human MCF-7 cells & Ray et al. (2007) & $\downarrow$ by Leptin \\
\hline & $\downarrow$ by Leptin & Human MDA-MB-361 cells & Ray et al. (2007) & \\
\hline
\end{tabular}

\section{References}

Bendinelli P, Maroni P, Pecori GF \& Piccoletti R 2000 Leptin activates Stat3, Stat1 and AP-1 in mouse adipose tissue. Molecular and Cellular Endocrinology 16811-20.

Cauzac M, Czuba D, Girard J \& Hauguel-de MS 2003 Transduction of leptin growth signals in placental cells is independent of JAK-STAT activation. Placenta 24 378-384.

Lee CI, Guh JY, Chen HC, Lin KH, Yang YL, Hung WC, Lai YH \& Chuang LY 2004 Leptin and connective tissue growth factor in advanced glycation end-product-induced effects in NRK-49F cells. Journal of Cellular Biochemistry 93 940-950.

Nakatsuji T 2006 Rat thymectomy effects on leptin receptor and T-bet: erythroid hyperplasia with maturation arrest and suppressed T-cellmediated hepatotoxicity. Laboratory Hematology 12 2-10.
Ray A, Nkhata KJ \& Cleary MP 2007 Effects of leptin on human breast cancer cell lines in relationship to estrogen receptor and HER 2 status. International Journal of Oncology 30 1499-1509.

De Rosa V, Procaccini C, Cava AL, Chieffi P, Nicoletti GF, Fontana S, Zappacosta S \& Matarese G 2006 Leptin neutralization interferes with pathogenic $\mathrm{T}$ cell autoreactivity in autoimmune encephalomyelitis. Journal of Clinical Investigation 116 447-455.

Smith JT, Acohido BV, Clifton DK \& Steiner RA 2006 KiSS-1 neurones are direct targets for leptin in the ob/ob mouse. Journal of Neuroendocrinology 18 298-303.

Uchida T, Nakamura T, Hashimoto N, Matsuda T, Kotani K, Sakaue H, Kido Y, Hayashi Y, Nakayama KI, Morris FW et al. 2005 Deletion of Cdkn1b ameliorates hyperglycemia by maintaining compensatory hyperinsulinemia in diabetic mice. Nature Medicine 11 175-182. 